\title{
Promoting Precision Addiction Management (PAM) to Combat the Global Opioid Crisis
}

\begin{abstract}
Kenneth Blum ${ }^{* 1-9}$, Edward J Modestino ${ }^{10}$, Jennifer Neary ${ }^{5}$, Marjorie C Gondré-Lewis ${ }^{8,11,12}$, David Siwicki ${ }^{5}$ Mark Moran $^{5}$, Mary Hauser ${ }^{4}$, Eric R Braverman ${ }^{5}$, David Baron ${ }^{3}$, Bruce Steinberg ${ }^{10}$, Thomas Mc Laughlin ${ }^{13}$ and Rajendra D Badgaiyan ${ }^{14}$
\end{abstract}

${ }^{1}$ Department of Psychiatry, Wright State University, USA

${ }^{2}$ Department of Psychiatry, University of Florida College of Medicine, USA

${ }^{3}$ Department of Psychiatry and Behavioral Sciences, Keck Medicine University of Southern California, USA

${ }^{4}$ Division of Applied Clinical Research \& Education, Dominion Diagnostics, USA

${ }^{5}$ Department of Precision Medicine, Geneus Health LLC, USA

${ }^{6}$ Department of Clinical Neurology, Path Foundation, USA

${ }^{7}$ Institute of Psychology, Eötvös Loránd University, USA

${ }^{8}$ National Human Genome Center at Howard University, USA

${ }^{9}$ Department of Psychiatry, University of Vermont, USA

${ }^{10}$ Department of Psychology, Curry College, USA

${ }^{11}$ Department of Anatomy, Howard University College of Medicine, USA

${ }^{12}$ Department of Psychiatry and Behavioral Sciences, Howard University College of Medicine, USA

${ }^{13}$ Center for Psychiatric Medicine, USA

${ }^{14}$ Department of Psychiatry, Icahn School of Medicine, USA

Received: January 24, 2018; Published: February 07, 2018

*Corresponding author: Kenneth Blum, Department of Psychiatry, Boonshoft School of Medicine, Dayton VA Medical Center, Wright State University, Dayton, OH, USA, Email: drd2gene@gmail.com

\section{Abstract}

It is universally agreed that dopamine is a major neurotransmitter in terms of reward dependence, however, there remains controversy regarding how to modulate its role clinically to treat and prevent relapse for both substance and non-substance-related addictive behaviors. It is also agreed by most that there is a need to provide early genetic identification possibly through a novel researched technology referred to Genetic Addiction Risk Score(GARS). ${ }^{\mathrm{TM}}$ The existing FDA-approved medications promote blocking dopamine, however, we argue that a more prudent paradigm shift should be biphasic-short-term blockade and long-term upregulation, enhancing functional connectivity of brain reward. It is critical to understand that the real phenotype is not any specific drug or non -drug addictive behavior, but instead is Reward Deficiency Syndrome (RDS). Thus the true phenotype of all addictive behaviors is indeed RDS. Finally, we are suggesting that one way to combat the current out of control Opioid /Alcohol crisis worldwide is to seriously reconsider treating RDS by simply supplying powerful narcotic agents (e.g. Buprenorphine). This type of treatment will only keep people addicted. A more reasonable solution involving genetic testing, urine drug screens using Comprehensive Analysis of Reported Drugs (CARD) and dopamine homeostasis we call " Precision Addiction Management" тM seems parsonomiuos.

Keywords: Genetic addiction risk score; Hypodopaminergic; Pro-dopamine regulation; Reward deficiency syndrome

Abbreviations: GARS: Genetic Addiction Risk Score; CARD: Comprehensive Analysis of Reported Drugs; ASI: Addiction Severity Index; BRC: Brain Reward Cascade; RDS: Reward Deficiency Syndrome; SUD: Substance Use Disorder; NAc: Nucleus Accumbens

\section{Introduction}

Following 27 years of extensive research by many scientists worldwide, a panel of ten reward gene risk variants, called the
Genetic Addiction Risk Score (GARS), has been developed. In unpublished work, when GARS was compared to the Addiction 
Severity Index (ASI), which has been used in many clinical settings, GARS significantly predicted the severity of both alcohol and drug dependency. In support of early testing for addiction and other RDS subtypes, parents caught up in the current demographic of over 100 people, both young and old, dying daily from opiate/opioid overdose, need help. In the past, families would have never guessed that their loved ones would die or could be in real danger due to opiate addiction. Insightful reporting by author, Bill Moyers, in Parade Magazine in the 90's, reported that as he traveled around the United States, he found many children with ADHD and other spectrum disorders like Autism, and noted that many of these children had related conditions like substance abuse. He called for better ways to identify these children and treat them with approaches other than addictive pharmaceuticals.

To our knowledge, GARS is the only panel of genes with established polymorphisms reflecting the Brain Reward Cascade (BRC), which has been correlated with the ASI-MV alcohol and drug risk severity score.

While other studies are required to confirm and extend the GARS test to include other genes and polymorphisms that associate with a hypodopaminergic trait, these results provide clinicians with a non-invasive genetic test. Genomic testing, such as GARS, can improve clinical interactions and decision-making. Knowledge of precise polymorphic associations can help in the attenuation of guilt and denial, corroboration of family genograms; assistance in risk-severity-based decisions about appropriate therapies, including pain medications and risk for addiction; choice of the appropriate level of care placement (i.e., inpatient, outpatient,

Table 1: Summary of KB220 variants in various treatment cohorts. intensive outpatient, residential); determination of the length of stay in treatment; determination of genetic severity-based relapse and recovery liability and vulnerability; determination of pharmacokinetic medical monitoring for better clinical outcomes (e.g., the A1 allele of the DRD2 gene reduces the binding to opioid receptors in the brain, thus, reducing naltrexone's clinical effectiveness); and supporting medical necessity for insurance scrutiny.

Moreover, Blum's laboratory following a 50 year sojourn developed pro-dopamine regulatory neuro-nutrients [see total cohort of patients studied over 37 clinical trials (Table 1) that enable real personalized treatment for all behavioral addictions following GARS testing. The interaction of genes and neurotransmitters control the release of dopamine is the Brain Reward Cascade (BRC) [1]. Variations in the BRC, possibly genetic or epigenetic, may predispose individuals to addictive behaviors and altered pain tolerance [2]. As mentioned above, the Genetic Addiction Risk Score (GARS), which is the first test which accurately predicts vulnerability to pain, and also addiction, and various other compulsive behaviors, within the umbrella of Reward Deficiency Syndrome (RDS), provides benefits for individuals suffering from Substance Use Disorder (SUD). Innovative strategies to combat epidemic opioid, iatrogenic prescription drug abuse, and death, based on the role of dopaminergic tone in pain pathways, have been previously proposed [3]. Sensitivity to pain may reside in the mesolimbic projection system, where genetic polymorphisms associate with a predisposition to pain vulnerability or tolerance [4].

\begin{tabular}{|c|c|c|}
\hline KB220 Variants* & Controls** & Anti- Addictive Behaviors*** \\
\hline 53 & 1010 & Withdrawal, Stress, Low Energy, Craving, Relapse, Low resting State Functional Connectivity l \\
\hline 708 & 49 & Withdrawal, Craving, Anxiety, Anhedonia, Relapse, AMA rate \\
\hline 50 & 42 & Drug Hunger, AMA rate, Withdrawal, relapse, regulated brain waves \\
\hline 353 & 181 & Withdrawal, Stress, Low Energy, Craving, Relapse, Anger, Fatigue \\
\hline 300 & 162 & Binge Eating, Sugar Craving, Weight Gain, High BMI, High Body Fat, Overeating, Late night eating, Lucid Dreams \\
\hline
\end{tabular}

Total *Experimental =1,464; Total ${ }^{* *}$ Controls $=1444$

${ }^{* * *}$ comparative statistics reveal between restore Gen and controls for all anti-addictive behaviors at least $\mathrm{P}<.05$ in a total of 35 studies.

These polymorphisms provide specific targets to assist in the treatment of pain and identify risk for subsequent addiction. Pharmacogenomic testing of candidate genes like DRD 1,2,3,4; MOA-A; COMT; DAT1; 5HTTLLR; OPRM and GABRA3 might result in pharmacogenomic, personalized solutions, and improved clinical outcomes. The genetically identifying risk RDS behaviors, especially within compromised populations, may be a frontline tool to assist municipalities in providing better resource allocation. The interaction of at least four neurochemical pathways: serotonergic,
GABAergic, endorphinergic and dopaminergic-together constitute the "brain reward cascade" (Figure 1) which is a natural sequence of events that produce feelings of well-being. These events including synthesis, vesicle storage, metabolism, release, and function of neurochemistry are regulated by genes, and their expression, regarding messenger RNA-directed proteins. Therefore, genetic testing can provide a window that may be utilized to identify an individual's neurochemistry and thus to formulate the optimal treatment options for them [5]. 


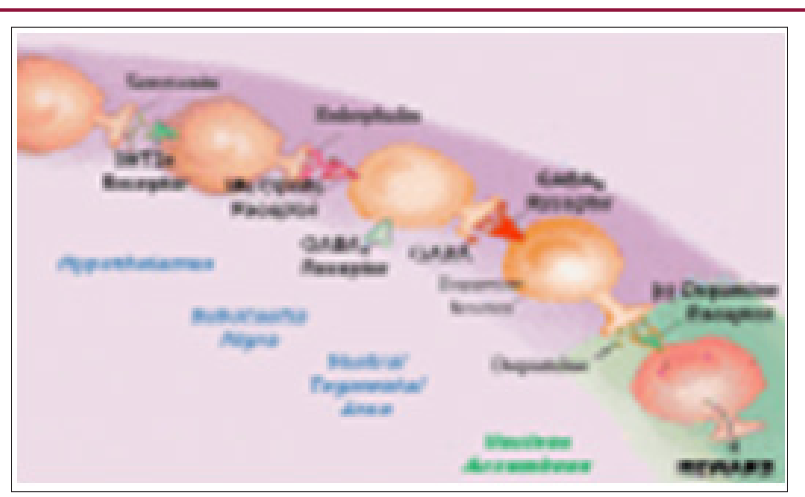

Figure 1: Brain Reward Cascade [6,7], In this cascade, the stimulation of the serotonergic system within the hypothalamus, through innervation, stimulates delta/mu receptors via the release of enkephalin. Further activation of the enkephalinergic system leads to inhibition of GABA transmission in the striatum via enkephalin stimulation of mu receptors upon GABA neurons. This inhibition allows the fine-tuning of GABA activity. Additionally, this provides the normal release of dopamine at the projected area of the NAc. Dopamine is a neurotransmitter with multiple essential functions including behavioral effects such as "pleasure" and "stress reduction." Without the typical function of dopamine, an individual will suffer from cravings and may have difficulty coping with stress.

Therefore, a hypodopaminergic brain genetically predisposes people to seek out substances and/or behaviors that can be used to overcome this craving state, whereby activating mesolimbic brain dopaminergic centers [8,9]. Psychoactive substances like alcohol, psychostimulants, and opiates, and risky behaviors like gambling, overeating, and thrill-seeking [10] induce the release of dopamine in synapse at the NAc, to overcome the hypodopaminergic state of that individual. Temporary relief from the discomfort and a false sense of well-being is the product of this self-medication [11]. Chronic abuse of substances often leads to inactivation, or a down regulation, e.g., inhibition of neurotransmitter synthesis, neurotransmitter depletion, a formation of toxic levels of neurotransmitters. Thus, pathological and substance-seeking behaviors are employed to provide a pleasurable response to decrease uncontrollable cravings.

For those Individuals with reward gene polymorphisms/ variations, given environmental insult, will be at risk for compulsive, impulsive, and addictive behavior. Reward Deficiency Syndrome (RDS) is a spectrum which includes and characterizes these genetically induced behaviors [12]. These pathological behaviors, which can consist of drug-abuse, are candidates for addiction including tolerance and dependence. The behavior or drug of choice by the individual is a function of both genes and environmental factors like availability and peer pressure. Previously we published on the potential of GARS to predict vulnerability or risk for both drug and alcohol severity as measured by the Addiction-Severity Index (ASI) [13]. However, a frequently raised question relates to what is the benefit of GARS ${ }^{\mathrm{TM}}$ testing in known addicts already in treatment programs. We believe that there are many important reasons for GARS testing in people expressing addictive behaviors of all types [14,15].

With regard to RDS, it is noteworthy that both substance and non-substance addictive behaviors (like music, food [glucose \& fat], sex. internet, gaming), cause a preferential release of dopamine in the nucleus accumbens [NAc], which suggests that people self-medicate to obtain their required dopamine fix. The acute release of dopamine leads to feelings of well-being in the very short term, especially in individuals who through either gene polymorphisms (variants), or gene/environment interactions (epigenetic-methylation, reduced expression) and or inhibition of deacetylation (increased expression) on histones in the chromatin material involving many genes affecting mRNA expression, have compromised dopaminergic function. This concept is clarified by the simple formula, whereby Phenotype $=$ Genetics + Environment $[\mathrm{P}=\mathrm{G}+\mathrm{E}]$. It is understood that this is a very complex interaction involving many polymorphic genes and their subsequent interaction with the environment (genes X environment).

It is important to be cautious to accept such genetic testing that uncovers reward circuitry gene polymorphisms, particularly those linked to dopaminergic pathways as well as opioid receptor(s) as a method of obtaining better treatment results. Comprehending the relationship between the reward circuitry's participation in buprenorphine outcomes and its corresponding genotypes deliver an innovative model to enhance a patient's clinical experience and improvements throughout opioid replacement therapy [16]. According to the American Society of Addiction Medicine (ASAM) they make a clear distinction between the concept of addiction and positive treatment outcomes related to buprenorphine not locking people into addiction but rather neuroadaptation which is arguable. However, both NIDA and NIAAA are desperately investigating an array of innovative ideas to help restore brain function especially hypodominergia, but we believe that our approach should be carefully considered and tested. In due respect we are encouraging scientist and clinicians respectively to at least embrace these simple concepts in the near future, and provide a new listen to the Blue Note All Stars record "Message of Hope to the many victims of overdose and death.

\section{Acknowledgement}

The authors appreciate the expert edits of Margaret A. Madigan and the superb assistance of Justin Jones and Erin Gallagher of Geneus Health, LLC. 


\section{References}

1. Blum K, Kozlowski GP (1990) Ethanol and neuromodulator interactions: a cascade model of reward. Alcohol and behavior. In: Ollat $\mathrm{H}$, Parvez S, Parvez H (Eds.). Alcohol and Behaviour: Basic and Clinical Aspects Progress in Alcohol Research. VSP International Science Publishers. Utrecht, Netherlands, 131-149.

2. Chen AL, Chen TJ, Waite RL, Reinking J, Tung HL, et al. (2009) Hypothesizing that brain reward circuitry genes are genetic antecedents of pain sensitivity and critical diagnostic and pharmacogenomic treatment targets for chronic pain conditions. Med Hypotheses 72: 1422.

3. Blum K, Oscar-Berman M, Dinubile N, Giordano J, Braverman ER, et al. (2013) Coupling Genetic Addiction Risk Score (GARS) with Electrotherapy: Fighting Iatrogenic Opioid Dependence. J Addict Res Ther 4: 1000163

4. Blum K, Oscar-Berman M, Jacobs W, McLaughlin T, Gold MS J (2014) Buprenorphine Response as a Function of Neurogenetic Polymorphic Antecedents: Can Dopamine Genes Affect Clinical Outcomes in Reward Deficiency Syndrome (RDS)? J Addict Res Ther 5: 1000185.

5. Blum K, Badgaiyan RD, Agan G, Fratantonio J, Simpatico T, et al. (2015) Molecular Genetic Testing in Reward Deficiency Syndrome (RDS): Facts and Fiction. J Reward Defic Syndr 1(1): 65-68.

6. Blum K (2012) Chapter 26: neurogenetics and nutrigenomics of reward deficiency syndrome. In: Barh D, Blum K, Madigan M (Eds.). Boca Raton: CRC J Addict Res Ther 3(5): 139.

7. Blum K, Oscar-Berman M, Stuller E, Miller D, Giordano J, et al. (2012) Neurogenetics and nutrigenomics of neuro-nutrient therapy for reward deficiency syndrome (RDS): clinical ramifications as a function of molecular neurobiological mechanisms. J Addict Res Ther 3: 139.

This work is licensed under Creative Commons Attribution 4.0 License

Submission Link: http://biomedres.us/submit-manuscript.php
8. Conner BT, Hellemann GS, Ritchie TL, Noble EP (2010) Genetic, personality, and environmental predictors of drug use in adolescents. J Subst Abus Treat 38: 178-190.

9. Comings DE, Blum K (2000) Reward deficiency syndrome: genetic aspects of behavioral disorders. Prog Brain Res 126: 325-341.

10. Dackis CA, Gold MS (1985) New concepts in cocaine addiction: the dopamine depletion hypothesis. Neurosci Biobehav Rev 9: 469-477.

11. Gold MS, Redmond DE, Kleber HD (1978) Clonidine blocks acute opiatewithdrawal symptoms. Lancet 2: 599-602.

12. Blum K, Chen AL, Oscar-Berman M, Chen TJ, Lubar J, et al. (2011) Generational association studies of dopaminergic genes in reward deficiency syndrome (RDS) subjects: selecting appropriate phenotypes for reward dependence behaviors. Int J Environ Res Public Health 8: 4425-4459.

13. Blum K, Chen ALC, Thanos PK, Febo M, Demetrovics Z, et al. (2018) Genetic addiction risk score (GARS) ${ }^{\mathrm{TM}}$, a predictor of vulnerability to opioid dependence. In: Front Biosci (Elite Ed) 1: 175-196.

14. Gumbley SJ (2016) Recovery in the 21st Century: From Shame to Strength. See comment in PubMed Commons bel J Addict Nurs 27: 143147.

15. Blum K, Modestino EJ, Gondré-Lewis MC, Chapman EJ, Neary J, et al. (2017) The Benefits of Genetic Addiction Risk Score (GARS ${ }^{\text {TM}}$ ) Testing in Substance Use Disorder (SUD). Int J Genom Data Min: IJGD p.115.

16. Blum K, Thompson B, Demotrovics Z, Femino J, Giordano J, et al. (2015) The Molecular Neurobiology of Twelve Steps Program \& Fellowship: Connecting the Dots for Recovery. J Reward Defic Syndr 1: 46-64.

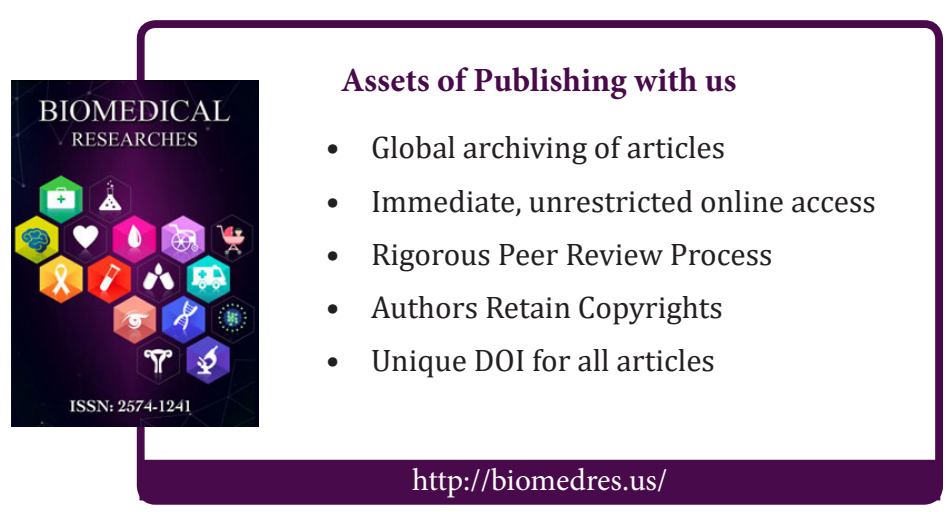

